\title{
Review
}

\section{Wak Engineering Evolution}

\author{
Paolo Armignacco Francesco Garzotto Mauro Neri Anna Lorenzin \\ Claudio Ronco
}

International Renal Research Institute of Vicenza (IRRIV), Department of Nephrology, San Bortolo Hospital, Vicenza, Italy

\section{Key Words}

Wearable artificial kidney · WAK latest developments · Miniaturization · Pumps for extracorporeal circulation

\begin{abstract}
The wearable artificial kidney (WAK) is a device that allows prolonged and frequent dialysis treatments for patients with end stage renal disease. It potentially provides a practical and feasible solution to satisfy the need for an optimal delivered dialysis. Until now, the WAK has already been presented in several models, proposing different therapeutic modalities and engineering approaches. The ideal solution of a WAK may come from a combination of all the prototypes developed and/or those currently in development. Consequently, it is important to have a complete overview and a deep knowledge of the possible engineering solutions in order to achieve the incremental steps necessary to solve the remaining issues of wearable extracorporeal blood purification devices. Since technical advances are continuously evolving and it is important to focus on clinical requirements and needs, a multidisciplinary collaboration has to be coordinated to achieve the final objective of the practical realization of a wearable artificial kidney. In such a context, the main aim of this article was to analyze more in detail the WAK Engineeristic Development.

(c) 2015 S. Karger AG, Basel
\end{abstract}

(c) 2015 S. Karger AG, Basel

0253-5068/15/0393-0110\$39.50/0

\section{The Wak: What it Is and its Purposes}

Although epidemiological data are contradicting and controversial, it is a well-known fact that end-stage renal disease (ESRD) is a considerable social and economic problem. Worldwide, both the incidence and prevalence of treated ESRD are increasing. Data on ESRD are quite extensive due to the availability of registries covering a large percentage of patients on renal replacement therapy (RRT) $[1,2]$. The demand for RRT in the treatment of ESRD is inexorably increasing, contributing to the inflation of healthcare spending [3].

Published evidence suggests that frequent and prolonged dialysis (daily dialysis) may improve health-related quality of life and potentially increase longevity of ESRD patients [4].

Despite the benefits of daily dialysis, its implementation using standard machinery is difficult for patients and the healthcare system in general. Wearable hemodialysis might represent an alternative by which more frequent treatments can be delivered to chronic kidney disease (CKD) patients with much less interference in their routines, thereby promoting better quality of life and allowing activities of daily living [5].

A WAK could be a very promising way to provide a practical solution to meet this need. The conceptualiza-

\section{KARGER 125}

E-Mail karger@karger.com www.karger.com/bpu
Prof. Claudio Ronco

Department of Nephrology, Dialysis and Transplantation

San Bortolo Hospital, The International Renal Research Institute

Viale Rodolfi 37, IT-36100 Vicenza (Italy)

E-Mail cronco@goldnet.it 
tion of WAK has been studied during the last decades, with different attempts in order to practically achieve this idea [6].

An ideal WAK should be wearable, comfortable, as well as lightweight. It also has to be highly efficient since it should operate like a real kidney, primarily allowing the necessary filtration rate as well as the maintenance of the acid-base balance and of the hydro-electrolytic equilibrium [6]. Recently, new approaches for miniaturization and transportability of medical devices have been developed, paving the way for wearability and the possibility of implantation for renal replacement therapies [7].

At present, there are a lot of technical proposals that have tried and are trying to achieve a practical realization of WAK system to be used in clinical practice.

\section{WAK Conceptualizations}

- Currently, the WAK has been conceptualized in several ways:

- ViWAK (Vicenza Wearable Artificial Kidney)

- AWAK (Automated Wearable Artificial Kidney)

- BioRAD (Bio-artificial Renal Assist Device)

- HNF (Human Nephron Filter)

- WAKMAN

- NEPHRON+

\section{ViWAK}

The Vicenza Wearable Artificial Kidney (ViWAK) [8] consists of a wearable system for enabling continuous ambulatory peritoneal dialysis in CKD patients.

It is a wearable system consisting of: (a) a double-lumen peritoneal catheter; (b) a dialysate outflow line; (c) a miniaturized rotary pump; (d) a circuit for dialysate regeneration featuring a waterproof container with 4 cartridges in parallel with a mixture of activated carbon and polystyrene resins; (e) a filter for deaeration and microbiological safety; (f) a dialysate inflow line, and (g) a handheld computer to function as a remote control.

The device weighs $2 \mathrm{~kg}$ and is connected to the patient using a double-lumen peritoneal dialysis catheter. Two liters of fresh PD solution first enter the peritoneal cavity. After $2 \mathrm{~h}$ when dialysate/plasma equilibration is approximately $50 \%$, the exhausted PD solution is being regenerated by adsorption on 2 packs, containing the 4 cartridges at a rate of $20 \mathrm{ml} / \mathrm{min}$ and recirculation is activated for a total of $10 \mathrm{~h}$. At the end, the liquid is drained and a 21 of icodextrin exchange is performed. The device provides a creatinine and a $\beta-2$ microglobulin clearance of 12-14 1/day equal to a weekly clearance of 70-90 1/week.

The ViWAK has the potential advantage of a reduced quantity of needed volume of dialysate that allows the optimization of the lightweight factor and the size of the device. On the contrary, since it is based on a peritoneal dialysis (PD) approach, the infusion of glucose and the instability of dialysate composition and $\mathrm{pH}$ might represent an obstacle for its application in clinical practice.

\section{AWAK}

The Automated Wearable Artificial Kidney (AWAK) [9] is a device suitable for any patient with ESRD who can receive peritoneal dialysis.

With such a device, patients will continue to have dialysate fluid sent into their abdomen. The primary and most important difference with conventional peritoneal dialysis is that patients who are treated with AWAK do not have to reestablish the dialysate regularly, since it is continuously regenerated with the AWAK technology and, as a consequence, it can be replaced once every 8-12 weeks. Spent dialysate is first passed through a hemofilter to produce a protein-free ultrafiltrate, which is then passed through a conventional sorbent cartridge and reconstituted with potassium, calcium, magnesium and glucose.

A peritoneal dialysis-based AWAK has the advantages of requiring no extracorporeal blood circulation (therefore it can be defined 'bloodless'), a limited dialysate volume and a reduced protein loss with respect to other technologies. Since it is based on PD therapy, the AWAK shows the same limitations of ViWAK regarding the use of glucose and the maintenance of $\mathrm{pH}$.

\section{BioRAD}

Humes et al. [10] suggested the concept of a Bio-artificial Renal Assist Device (BioRAD) to replace not only filtrative but also metabolic and endocrine functions of the kidney.

BioRAD is composed of a hemofilter and hollow-fiber cartridges containing human tubular cells derived from donor organs unsuitable for human transplantation: they consist of renal proximal tubule cells grown as confluent 
monolayers of a multifiber bioreactor with a membrane surface area from 0.4 to $1.6 \mathrm{~m}^{2}$. Cells along the inner surface of hollow fibers are immunoprotected from the patient's blood by the hollow fiber membrane.

BioRAD has demonstrated promising results in clinical trials in acute kidney failure patients [11]. In particular, this device has been shown to tolerate a uremic environment while providing reabsorptive, metabolic, and endocrine activity.

However, since this device has been clinically tested only in comparison to an existing hemofiltration system, more trials are to be performed with it so that it can be considered really feasible.

\section{HNF}

The Human Nephron Filter (HNF) [12] utilizes the bottom-up nanotechnology that would at last make possible the realization of an implantable, wearable or continuously operating artificial kidney.

This device is made up of two membranes that operate in series inside one device cartridge. The first membrane simulates the glomerular function, with the use of convective transport generating a ultra-filtrate, which contains all solutes approaching molecular weight of albumin $(65 \mathrm{kDa})$. On the other hand, the second membrane simulates renal tubules function, selectively reclaiming preidentified solutes to maintain body homeostasis.

In this device, no dialysis solution is used, thereby allowing a reduction in weight and encumbrance. The HNF has been computer modeled and, operating $12 \mathrm{~h}$ /day, 7 days/week, it provides the equivalent of $30 \mathrm{ml} / \mathrm{min}$ glomerular filtration rate, the value that has been proposed as target clearance for a general WAK [13]. The HNF system, through dialysate elimination and the use of a novel membrane system created through applied nanotechnology, is a breakthrough in RRT based on the function of native kidneys. Modeling studies have demonstrated lower time average urea concentration in a human nephron filter-modeled patient compared with conventional hemodialysis [14].

\section{WAKMAN}

WAKMAN is a wearable/portable ultrafiltration system used in overhydration and congestive heart failure therapy [15]. The WAKMAN consists of a wearable high-tech vest incorporating a miniaturized blood filtra- tion system made of blood pump and hemofilter; the unit is so compact and lightweight that it can be carried in a pocket (with a size similar to an old style music 'walkman'). Patients can wear the vest and connect the intravascular catheter to the circuit through which blood flows, gets ultrafiltered by the filter and returns to the patient.

The possible use of piezoelectric components in the blood pumping system of the WAKMAN can be a very promising solution as an alternative to conventional peristaltic pumps and their related issues (e.g., high power consumption, hemolysis) [16]. However, this technology has to be optimized to satisfy the several specifications (clinical and technical) required for an extracorporeal blood propelling system.

The last evolution of WAKMAN device is equipped with oscillating displacement pumps, specifically designed for propelling non-Newtonian fluids. An elastic diaphragm, coupled with a non-return valve, is stressed by an internal axle moved by a DC motor, allowing onedirectional blood flow. Despite the several potential advantages of this technology, problems regarding biocompatibility still have to be completely solved.

\section{NEPHRON+}

As a project of the Framework Programme (FP7) for research and ICT technological development, WAK $(\mathrm{NEPHRON}+)$ [17] includes a prototype of artificial kidney with a target dimension of $10 \times 6 \times 4 \mathrm{~cm}$ and weighing less than $2 \mathrm{~kg}$. Based on fractionated plasma separation and adsorption, a high flux filter separates blood from plasma, which is purified by nanostructured sorbents and then returned to the blood; hence, no dialysate fluid is needed.

\section{The Current 'WAK'}

Actually, what is properly called a wearable artificial kidney (WAK) is the belt-type wearable HD machine developed by Gura and colleagues [18].

Its working principle is based on drawing blood from a double lumen catheter, anticoagulating it with heparin from a reservoir using a micro pump and then circulating it through the blood channel of the WAK shuttle pump and into the dialyzer. The blood then returns to the venous side of the double lumen catheter, and clean dialysate enters the dialyzer. The dialysate circulates in coun- 
tercurrent flow to the blood and exits into the dialysate channel of the WAK pump. Another pump removes a predetermined amount of the spent dialysate into a collection bag. The dialysate then returns to the dialyzer after going through a series of sorbents and being infused with a solution containing sodium bicarbonate.

\section{Ideal WAK}

Probably, nowadays the device of Gura and colleagues is the one that realistically better reflects the ideal conceptualization of a WAK.

In order to achieve a true wearability, the WAK has to face and solve some challenges, such as those related to its safety, ergonomics, lightweight but powerful battery design, clotting avoidance and limited toxicity. The flexible design should be positioned on the user to mimic an operating kidney and should be discrete and comfortable to allow the patient to move freely.

The requirements for new technologies in dialysis, in effect, are based on the following objectives:

- Safety;

- Biocompatibility;

- Ease of use;

- Dependability (a system is defined dependable if it is reliable, available, safe, confidential, maintainable and integral);

- Continuous power supply;

- Portability or even better implantability;

- Limited mechanical noise;

- Elimination of molecular weight solutes similar to kidney function.

The weight, shape and form of the WAK have to allow ergonomic adaptation to the body contour so that it can be worn continuously and permit the patient to sleep, sit and walk comfortably. Additionally, it should be easy to use and dependable, so that it can be used without the frequent need of expert assistance. Freedom from an electrical connection is required; yet there is the need for sufficient energy to propel blood and dialysate through the device. This means that batteries should be light but still deliver enough power. Highly efficient pumps should be used.

\section{Requirements for Pumping System}

One of the most critical and important components of a wearable device performing an RRT is certainly its pumping system. Many of the WAK's most important characteristics are strictly related to this pumping system. One of the main problems in WAK development and use is the one that is related to its pumping system integration, both in the device and in the interaction with the patient.

In fact, the ideal WAK pumping system should mainly allow:

- high safety;

- good biocompatibility properties;

- low hemolysis;

- high precision and accuracy;

- low power consumption;

- dimensions, weight and costs of production as low as possible.

What is more important is that its ergonomics and resistance (dynamic, static, thermal, usury) should be optimized.

\section{Future Challenges}

Nowadays, one of the key words in medical service is personal healthcare or patient-specific/tailored diagnosis/treatment. The evolution of the artificial kidney has followed this paradigm and many studies have predicted the future direction of RRT for chronic patients: personalization and daily treatment. To achieve these main aims, technological development has to reach its maximum potential in order to satisfy the clinical need of a personalized dialysis therapy.

During the last decades, major attempts and remarkable technological advances in the field of portable and wearable WAK systems have been accomplished. At this time, the conceptualization of WAK has already passed the feasibility phase and is now in the phase of the search for reliability.

However, the future development of a device, such as the WAK, is strictly related to a few technical issues that have to be reconsidered and analyzed together with social, ethical, legal and economic aspects. In particular, the development of an adequate pumping system both for blood and other fluids (in light of the requirements written earlier), a safe vascular access that may guarantee at the same time a certain mobility to the patient, a general low power consumption of all the components should be operative in a WAK device. To resolve these technical issues efficiently, professionals in the biomedical field have to work together: engineers must collaborate with physicians, nurses, biologists, economists and social scientists, in order to face the problem and the consequent technical requirements from a multidisciplinary point of view. 


\section{References}

$>1$ Meguid El Nahas A, Bello AK: Chronic kidney disease: the global challenge. Lancet 2005; 365:331-340.

$>2$ Environmental health: Gold, mercury and health. Wkly Epidemiol Rec 1994;69:275278.

$>3$ Winearls CG, Haynes R, Glassock R: CKD staging - evolution not revolution. Nefrologia 2010;30:493-500.

-4 Lee CP, Zenios SA, Chertow GM: Cost-effectiveness of frequent in-center hemodialysis. J Am Soc Nephrol 2008; 19:1792-1797.

$>5$ Kim JC, Ronco C: Current technological approaches for a wearable artificial kidney. Contrib Nephrol 2011;171:231-236.

$\checkmark 6 \mathrm{Kim}$ JC, et al: A wearable artificial kidney: technical requirements and potential solutions. Expert Rev Med Devices 2011;8:567579.
7 Fissell WH, Roy S, Davenport A: Achieving more frequent and longer dialysis for the majority: wearable dialysis and implantable artificial kidney devices. Kidney Int 2013;84:256264.

$>8$ Ronco C, Fecondini L: The Vicenza wearable artificial kidney for peritoneal dialysis (ViWAK PD). Blood Purif 2007;25:383-388.

$>9$ Lee DB, Roberts M: A peritoneal-based automated wearable artificial kidney. Clin Exp Nephrol 2008;12:171-180.

-10 Humes HD, et al: Tissue engineering of a bioartificial renal tubule assist device: in vitro transport and metabolic characteristics. Kidney Int 1999;55:2502-2514.

11 Humes HD, et al: Initial clinical results of the bioartificial kidney containing human cells in ICU patients with acute renal failure. Kidney Int 2004;66:1578-1588.

12 Nissenson AR: Bottom-up nanotechnology: the human nephron filter. Semin Dial 2009; 22:661-664.

13 Fissell WH, Roy S: The implantable artificial kidney. Semin Dial 2009;22:665-670. ter: toward a continuously functioning fimplantable artificial nephron system. Blood Purif 2005;23:269-274.

15 Ronco C, Davenport A, Gura V: The future of the artificial kidney: moving towards wearable and miniaturized devices. Nefrologia 2011;31:9-16.

16 Kang J, Scholz T, Weaver JD, Ku DN, Rosen DW: Pump design for a portable renal replacement system. J Med Devices 2011;5:031008.

17 http://www.nephronplus.eu/en.

18 Gura V, et al: Continuous renal replacement therapy for end-stage renal disease. The wearable artificial kidney (WAK). Contrib Nephrol 2005;149:325-333.

19 Gura V, et al: Technical breakthroughs in the wearable artificial kidney (WAK). Clin J Am Soc Nephrol 2009;4:1441-1448. 